\title{
Development of a Non-contact Infrared Thermometer
}

\author{
Jing Zhang \\ School of Energy Engineering, Yulin University, Yulin,719000, China \\ zj_zhangjing@126.com
}

Keywords: Infrared temperature sensor; Thermometer; SCM; Alarm

\begin{abstract}
Due to the disadvantages of traditional mercury thermometers, such as longer measurement time and necessity of contact with the human body, a thermometer that uses infrared sensors to detect temperature without contact is designed. The Infrared temperature sensor MLX90614 is designed to collect human or object temperature by the SCM to process the temperature into the LCD display and alarm when over-temperature. Using software design to complete the control of the system. The smart thermometer can achieve non-contact measurement, place the thermometer in the forehead for a few seconds to get the body temperature, to alarm once the set value is exceeded. The design temperature range is $0-55{ }^{\circ} \mathrm{C}$, and temperature resolution is $0.1{ }^{\circ} \mathrm{C}$.
\end{abstract}

\section{Introduction}

Body temperature is the most basic and vital indicator of life. Measuring body temperature plays an important role in daily care. However, in the face of people's pursuit of a fast and safe lifestyle nowadays, the traditional mercury thermometer needs to measure about 5 minutes under the armpits and needs to be read by human eyes. Therefore, there are many drawbacks. With the development of infrared technology, infrared thermometers have also been recognized by the public due to their safety and rapidity. Infrared thermometer is mainly based on the principles of black body radiation to measure the human body's infrared radiation wavelength, followed by the measurement of body temperature, infrared sensors used by it only to absorb the infrared radiation of human body without any emission, which uses a passive non-contact measurement methods and can effectively prevent cross-infection of the human body, it is safe and convenient, so the infrared thermometer does not cause harm to the human body [1,2]. Compared with the traditional thermometer, the infrared thermometer is safe to use and has convenient measurement and short measuring time. Therefore, the research on infrared thermometer design has important theoretical and practical significance.

\section{Analysis of System Structure}

The design of the microcontroller-based controller, the Infrared temperature sensor directly to the collected signal for internal amplification and data processing and send it into the microcontroller, the microcontroller is to achieve LCD display and over-temperature alarm. The system diagram is shown in Fig. 1.

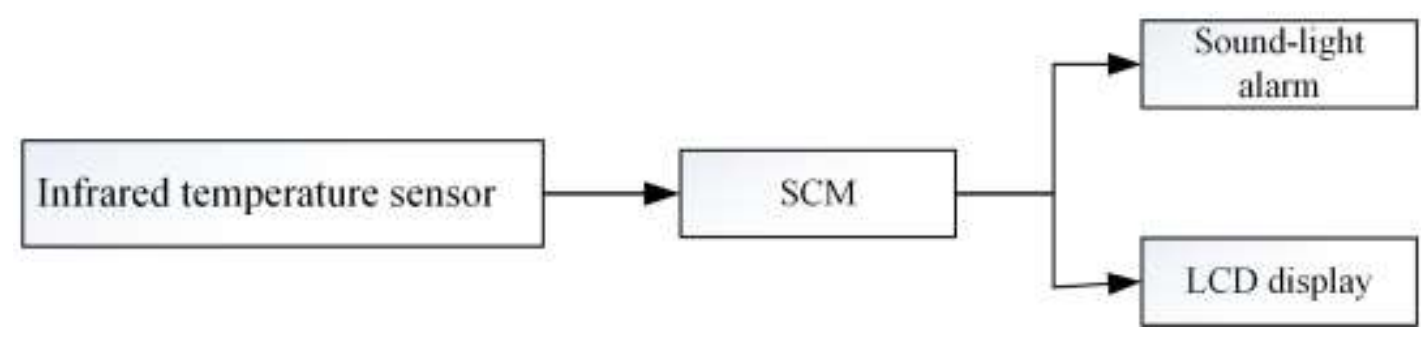

Figure 1. Block diagram of system 


\section{Hardware Circuit Design}

Temperature Measurement Circuit Design. MLX90614 is a non-contact infrared temperature measurement device, it uses low-noise amplifier, $17 \mathrm{~b}$ A/D converter and powerful DSP processing unit with high temperature resolution and measurement accuracy. The thermometer temperature range is $-40 \sim+125^{\circ} \mathrm{C}$, calculate and store in RAM ambient temperature and object temperature can achieve data with $0.01^{\circ} \mathrm{C}$ resolution. In addition, the sensor outputs digital signals that are accurate and linearly correlated with temperature, which simplifies the design. For the MLX90614, the accuracy is shown in Fig. 2 within the range of $10^{\circ} \mathrm{C}-40^{\circ} \mathrm{C}$ ambient temperature and $32^{\circ} \mathrm{C}-42^{\circ} \mathrm{C}$ tested object temperature $[3,4]$. Ta is the ambient temperature and To is the measured temperature. Since the thermometer is generally used indoors, the temperature usually ranges from 20 to $30{ }^{\circ} \mathrm{C}$ and the human body temperature ranges from 35 to $42{ }^{\circ} \mathrm{C}$. As shown in the Fig. 2, the accuracy of the MLX90614 is $0.1{ }^{\circ} \mathrm{C} \sim 0.2^{\circ} \mathrm{C}$, so the design uses MLX90614 infrared temperature sensor to meet the design requirements.

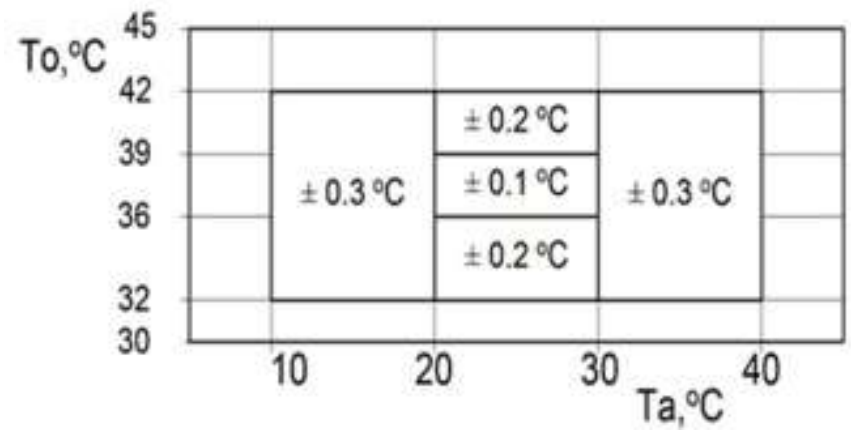

Figure 2. The accuracy of MLX90614 medicine

The working principle of infrared sensor MLX90614 is to transform the infrared radiation signal collected from objects and bodies into electrical signals, send the electrical signal into converter after noise amplification processing by amplifier, then the electrical signal is converted to digital signals and store the processed signals into the internal memory, finally send the signals into the SCM control system for further processing [5].

MLX90614 infrared temperature sensor uses the SPI bus, when connected to the microcontroller, SCL termination microcontroller serial input port RXD, serial output pulse signals of sensor are provided by the microcontroller, it is used to transmit temperature information, SDA serial output port TXD is used to provide temperature information for the microcontroller, in the specific operation, the microcontroller transfer data through the serial port mode 0 . Infrared temperature measurement circuit is shown in Fig. 3.

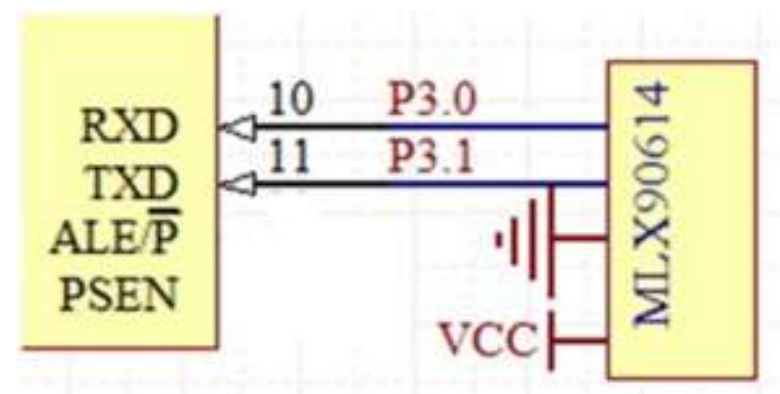

Figure 3. Infrared temperature measurement circuit

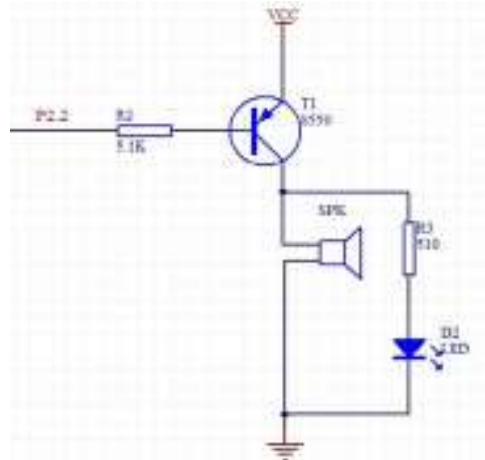

Figure 4. Alarm circuit

SCM. STC89C52RC microcontroller is adopted because it is a low-power, high-performance microcontroller. With flexible 8-bit CPU and in-system programmable Flash, it provides a highly flexible and efficient solution for a wide range of embedded control applications. The download 
speed is faster, at the same time in the download process, it can be downloaded directly at the serial port [6].

Alarm Circuit. Alarm circuit will make alarm when the body temperature exceeds the set value. The design uses active buzzer DC5V. When the system is powered on, the current drives the transistor T1 8550 to amplify to trigger the buzzer alarm through the current limiting resistor R2. The circuit is shown in Fig. 4.

Design of Display Circuit. The commonly used LCD1602 is adopted in this design, it can display two lines, there are 16 characters in each line, using a single $+5 \mathrm{~V}$ power supply, and its peripheral circuit configuration is simple, cheap, with a high cost performance[7]. The display circuit is shown in Fig. 5.

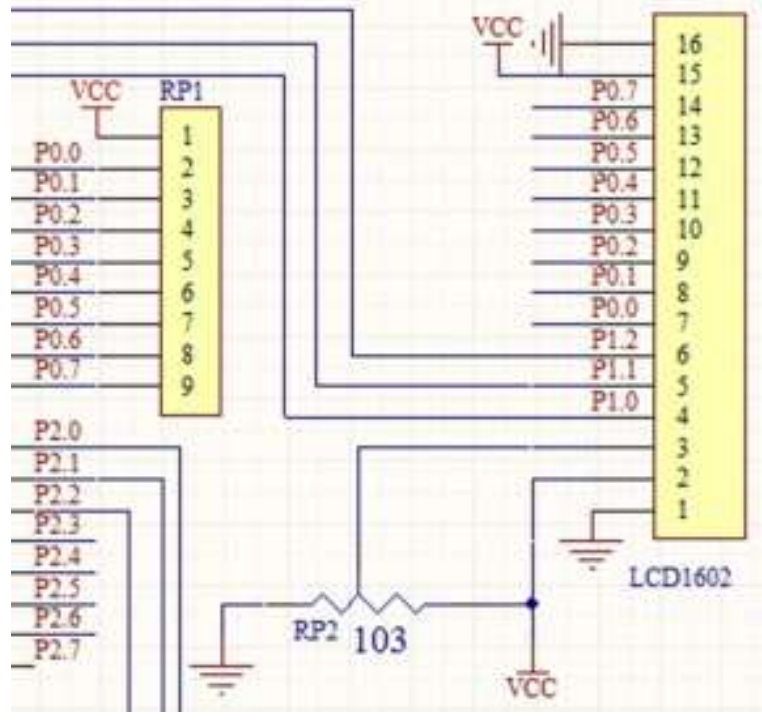

Figure 5. Display circuit

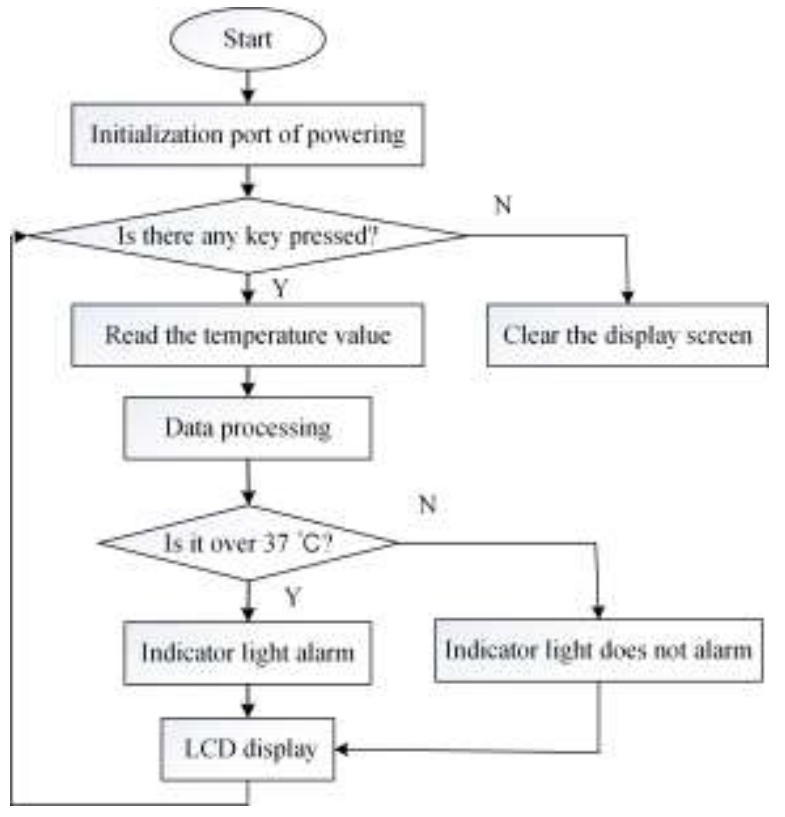

Figure 6. Main program flow chart

\section{Software Design}

The design of software program uses Keil uVsion4 compiler software, and the programming has four main parts, namely the main program, infrared temperature measurement program, the display program and alarm program. The main program work process is that the system is normally powered, start the initialization, when a key is pressed, the sensor will detect the signal transmitted to the microcontroller, the microcontroller is to process the data, send to the display and alarm, if there is no key press, the liquid crystal display is clear. The main program flow chart is shown in Fig. 6.

\section{Production and Commissioning}

The main design temperature measurement circuit, clock circuit, power circuit, reset circuit, display circuit, alarm circuit. Therefore, before the production of its overall layout of the first circuit layout, wiring, reduce cross-connection, the production is carefully carried out, after the completion of the production, with a multimeter to detect the connectivity of welding circuit. When circuit check is completed, debug the object, supply the power, press the start button to observe and record the ambient temperature value on LCD display, and then repeatedly measure the body temperature in different parts to compare and analyze the error. Fig. 7 is a figure of welded object.

Finally, to test different parts of the human body using the design of the infrared thermometer and the traditional thermometer, the statistical measurement results are shown in Table 1 
Table 1 Temperature Test Comparison Table

\begin{tabular}{|c|c|c|}
\hline & Infrared thermometer $\left[{ }^{\circ} \mathrm{C}\right]$ & Mercury thermometer $\left[{ }^{\circ} \mathrm{C}\right]$ \\
\hline Armpit & 36.7 & 36.9 \\
\hline Oral & 36.9 & 37 \\
\hline Ear & 36.5 & - \\
\hline Forehead & 36.5 & - \\
\hline
\end{tabular}

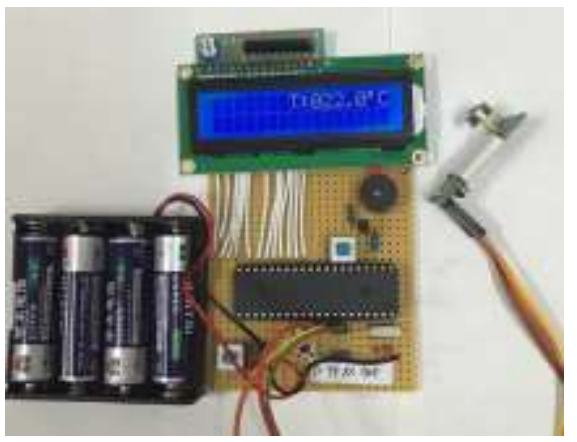

Figure 7. Infrared Thermometer

After comparison, through the temperature tests on different parts of the body measured by infrared thermometer and mercury thermometer, we can see that the results of infrared thermometer is small different with mercury thermometer, at the same time, temperature values of different parts of body are different. The general oral temperature is close to the body's temperature [8]. As can be seen from the table, the mercury thermometer is inconvenient to measure the temperature of ear, forehead and other external temperature. At the same time, in the testing process, there is a temperature difference in the infrared thermometer measurement, so it is recommended to use multiple measurements in the specific use and to take the average value, infrared thermometer is generally used for ear measurement.

\section{Analysis and Processing of System Error}

The developed infrared thermometer will cause some measurement error due to the following interferences, and the interferences are as follows $[9,10]$ :

Interference light. The system measures body temperature mainly by measuring the infrared radiation emitted by the object, so that all light outside the system may interfere with the system.

Ambient temperature. The higher the ambient temperature, the greater the impact on the system.

Measuring distance.

Measuring objects and time. The design is mainly aimed at the human body, the wavelength range of body's radiation is $7 \sim 14 \mathrm{um}$, the measured wavelength of system is close to the real value, but can not rule out the interference of other light or objects.

In order to avoid large errors, the following measures can be taken:

Multiple measurements to take the average value;

Select the best measuring distance between $2 \sim 4 \mathrm{~cm}$;

When measuring, select the site where the temperature is closest to human body and measure it under suitable environment;

\section{Conclusion}

Physiological parameter of body temperature is the most important and basic human life indicator, and infrared thermometer is a new type of non-contact thermometer. It has the fast response, safety and other characteristics compared with the mercury thermometer. At the same time, the over-temperature alarm, LCD display and other functions are designed to make it more perfect. 


\section{References}

[1] Luo Yaping, Design of data acquisition system based on LabVIEW for infrared thermometer ,Intelligence Detection.5(2014)52-56.

[2] Li Nana,Design of infrared temperature measurement system based on MLX90615 and MSP430,Journal of Sensors and Systems. 9(2011) 115-117.

[3] Lei Furong, 51 SCM common module design query manual,second ed., Tsinghua University Press, Beijing,2016.

[4] Liang Lianwei, Intelligent home security system, Sensor World.1(2013) 31-33.

[5] Li Hao, Infrared thermometer design using STM32F103 chip,World Electronics.9(2013) 45-56.

[6] Li Zhaoqing. Microcontroller theory and interface technology fourth ed., Beijing Aerospace University Press,Beijing, 2013.

[7] Zhou Peng,Design of temperature detection system based on STC89C52 microcontroller ,Electronic Technology. 22(2012):10-13.

[8] Qi Lei, Design of temperature control system based on AT89C52 microcontroller,Master,Inner Mongolia University, Hohhot ,China,2013.

[9] Wei Tanxun, Research and implementation of comprehensive error compensation technology non-contact temperature measurement, Master, Hangzhou University of Electronic Science and Technology ,Hangzhou,China, 2012.

[10]Luo Xiaojinc The calibration method of infrared thermometers for measurement of human temperature,Measurement and Testing Technology.7(2013)10-11. 